\title{
Alignment in a fish school: a mixed Lagrangian-Eulerian approach
}

\author{
M. Adioui, J.P. Treuil, O. Arino* \\ GEODES-IRD 32, avenue Henri Varagnat, F-93143 Bondy Cedex, France
}

Received 12 April 2001; received in revised form 17 January 2003; accepted 19 March 2003

\begin{abstract}
Social organization is one of the fundamental aspects of animal behavior, and has received attention both from experimental and theoretical perspectives. Examples of social groups appear at every size scale from the microscopic aggregates of mammalian cells (such as fibroblasts) to macroscopic herds of wildbeast, flocks of birds, and fish schools. There are two general frameworks when modeling such problems: the Lagrangian viewpoint and the Eulerian one. In this paper, we use both the approaches in the study of fish alignment. An individual-based model (IBM) (Lagrangian) provides a virtual world where fish forming a fish school try to adopt a common angular position. Fish are assumed to lie in horizontal planes, an individual angular position is the angle made by the oriented axis associated with the individual (tail to head) with a fixed direction. Two main forces are acting, a force of alignment, whose strength is assumed to be fixed in a given experiment but may be modified, and a force of dispersion, accounting for all disturbances. A transition from dispersion-dominant to alignment-dominant can be observed in the IBM experiments. A related PDE model (Eulerian) is used to determine the transition with sufficient accuracy.
\end{abstract}

(C) 2003 Published by Elsevier Science B.V.

Keywords: IBM; Lagrangian model; Eulerian model; Alignment; Schooling; Standard deviation; Stabilization time

\section{Introduction}

In many animal species, individuals aggregate to form temporary or permanent groups. Advantages of group formation have been demonstrated for a wide range of animals: it may improve food intake and growth, reduce vulnerability to predation risks that vary with fish size and environmental conditions, or even save energy (for example, through hydrodynamic effects in the case of fish grouping: Fréon and Misund, 1999; Parrish and Hamner, 1997; Stöcker, 1999). Our interest in this paper is in fish schools.

\footnotetext{
* Corresponding author. Tel.: +33-1-48-02-55-83; fax: +33-1-48-47-30-88.

E-mail address: arino@bondy.ird.fr (O. Arino).
}

A fish school is defined as the special moving condition of fish in which all individuals are oriented in a common direction, regularly spaced and moving at a uniform speed. The control of mutual separation distance involves individual forces acting on fish: forces of mutual attraction when the individuals are a sufficient distance from each other, and of repulsion when they come closer than a certain critical distance (Breder, 1959). Each fish reacts differently according to the range of distances in which a neighbor is positioned. Note that the mean distance to the nearest-neighbor (NND) varies among the species, between 0.3 and $3 \mathrm{BL}$ (BL: body length of fish) (Aoki, 1980; Huth and Wissel, 1994; Pitcher and Parrish, 1986). Each fish, having established its position, uses its eyes and its lateral line simultaneously 
to measure the speed of all the other fish in a school (Pitcher and Parrish, 1986). Aoki (1982) developed a simulation model in which schooling fish display the three behavioral interaction forces. Huth and Wissel (1994) used a model derived from Aoki to examine differences arising from different algorithms for balancing the effects of several neighbors. Warburton and Lazarus (1991) also compared different behavior algorithms of animal grouping.

Of the above three main forces implicated in fish schooling, we focus here on the alignment, that is the process by which an individual turns to adapt its orientation to that of its neighbors. During the last 20 years orientation processes of fish and animals have received much attention (Alt, 1997; Edelstein-Keshet and Ermentrout, 1990; Grunbaum, 1998; Grunbam and Okubo, 1994; Gueron and Levin, 1995; Gueron et al., 1996; Mogilner et al., 1999; Parrish and Hamner, 1997; Parrish and Edelstein-Keshet, 1999). One can think of many biological examples in which alignment to a common direction or a set of common directions occurs: in a flock of flying birds or a school of fish, individuals moving together as a group orient to the same direction of motion (Grunbaum, 1994; O'Brien, 1989). Alignment phenomena occur also in microscopic and in nonbiological systems (Mogilner et al., 1996). A discussion of alignment as a mechanism for enhancing chemotactic ability of social organisms such as schooling fish towards weak, noisy gradients is given in (Grunbaum, 1994). Locust swarms orient strongly in the direction of the wind (Mogilner et al., 1999). Flocks of migrating birds may use the earth magnetic field as a directional cue (Mogilner et al., 1999). On the microscopic scale, motile mammalian cells (such as fibroblasts) tend to align strongly on an artificial substrate (Mogilner et al., 1996). The degree of alignment of a group is sometimes expressed in terms of the polarization coefficient. Polarization takes value between 0 and $90^{\circ}$ (Huth and Wissel, 1994). As it approaches $0^{\circ}$, fish are nearer to heading in the same direction, while as polarization approaches $90^{\circ}$, fish are heading at right angles to one another. Huth and Wissel referred to these conditions, respectively, as "polarized" and "confused" (Huth and Wissel, 1994).

Alignment is a dynamical process and, as such, can be modeled according to two general frameworks, Eulerian and Lagrangian (Grunbam and Okubo, 1994). Eulerian models (also known as continuum or popula- tion models) deal with the time evolution of the distribution of animal density, described in most situations by partial (integro) differential or difference equations. The built-in mechanisms of such models do not generally include individual variability (Grunbaum, 1994).

In Lagrangian models (also known as individualbased models (IBMs)) the state of every single individual is given by a set of characteristic factors (location, age, etc.) and the time evolution of each factor is described by ordinary or stochastic differential equations (Niwa, 1996) or by a set of local rules (Deutsch, 1995; DeAngelis and Gross, 1992; Stöcker, 1999). In such models the trajectories of individual animals are calculated according to equations that may incorporate both physical laws (e.g. conservation of momentum) and elements of social behavior (such as attraction to, repulsion from, or tendency to align with neighbors). Fundamental to this approach is the recognition that individuals within a population are not like identical molecules; they differ from one another, and their differences have an effect on the behavior of the entire population. Some time ago already, Huston et al. (1988) anticipated the prospects of applying the individual-based approach using computers and the possibility of applying numerical simulations and numerical solutions. Indeed, numerical simulations flourished during the last 10 years; the results obtained are frequently in accordance with observations or real life experiments (Reynolds, 1999; Vabø and Nøttestad, 1997). However, mathematical tools are lacking for providing rigorous justification to such findings.

This contribution addresses the issue of fish alignment in a school using an IBM approach. Alignment here is meant as the nearing of the horizontal angular positions of the tail-to-head line of fish supposedly lying in horizontal planes. An IBM is described; its implementation on a computer allowed a large number of "virtual" experiments to be undertaken. Analysis based on these results is performed. The view taken here is that alignment is a dynamic process involving a phenomenological species-dependent strength of alignment competing with environmental noise. An important feature of our approach is that we work under the assumption of a sufficiently dense school and a time scale short enough for all movements inside the school, but alignment, to be negligible. In other words, we are specially concerned with the behavior of fish 
in the case when fish feel neither attraction toward nor repulsion from its neighbors. According to Huth and Wissel (1994), the wide range of this "neutral zone" is approximately $1.5 \mathrm{BL}$. It is natural to assume that the interactions have some finite (maximum) visual limiting distance, beyond which fish cannot perceive its neighbor. For the sake of simplicity, we omitted this assumption in the simulations; this point is discussed in the conclusion. Another simplifying assumption made in this work is that all fish in the school are in some sense equal. We did not take existence of a leader into account (Bumann et al., 1997; Krause et al., 2000).

The main results of this work are that the dynamics is controlled by a single parameter, that is the ratio, $\lambda$, of the strength to the noise. Simulations show that there is a threshold value such that when $\lambda$ is below this value, the noise dominates, while above it an alignment pattern starts to establish itself. In addition, the stabilization time was estimated, it was found that it decreases as $\lambda$ increases.

Estimating an accurate value for the threshold for alignment is not an easy task, especially when dealing with an IBM. A mathematical Eulerian model, which in fact arises from the IBM, was independently investigated and was used to help locate this threshold value more accurately. It also helped improve the IBM. A sketchy derivation of the passage from the IBM to the Eulerian model is included. A detailed mathematical analysis of the model can be found in Adioui et al. (in press). Finally, the validity of the approach followed in this paper, that is isolating the alignment from the other processes involved in fish schooling, is discussed in the conclusion: a time scale analysis performed there shows that it is justified if the polarization is high enough.

The paper is organized as follows. In Section 2, we describe the Eulerian aggregation model we just mentioned. It is in fact a very mild adaptation of a model that appeared earlier in the literature (Grunbaum, 1998). Some useful qualitative results are summarized from Adioui et al. (in press). The IBM is presented in Section 3: from generalities on the subject to the model used in the simulations of this paper. Section 4 is devoted to the results. Three different results are presented, corresponding to the following issues: (1) (the computation of) the threshold; (2) the time of stabilization; (3) the effect of density. Section 5 of- fers an extensive discussion, based in part on the comparison of the results with observed characteristic features.

The following general assumptions will be held throughout the paper. We deal with fish schools of rather small fish (sardine, anchovy, mackerel): the parameters that will be used on occasion are relevant to such sizes. Demography (birth and death) as well as growth are not considered.

\section{Eulerian alignment model}

In the Eulerian approach, the model describes fluxes of individuals through any given angular position $\theta$ (Grunbam and Okubo, 1994). Since the total population is constant, we may, and do, consider the proportion of individuals having a certain angular position as the state variable. For detailed explanations about the Eulerian model, we refer to Adioui et al. (in press). The distribution of angular positions, $U(\theta, t)$, evolves according to

$$
\frac{\partial}{\partial t} U(\theta, t)=d \frac{\partial^{2}}{\partial \theta^{2}} U(\theta, t)-\beta \frac{\partial}{\partial \theta}(U(\theta, t)[G \times U(\theta, t)]),
$$

where $d$ is the diffusion coefficient. We define the function $G \times U$ as the classical convolution of $U$ with the function $G$ given by

$G(\theta)= \begin{cases}1 & \text { on }]-\pi, 0](\bmod 2 \pi) \\ -1 & \text { otherwise }(\bmod 2 \pi) .\end{cases}$

The second member of the right-hand side of Eq. (1) is the analog of a gregarious effect, individuals tending to adopt the dominant orientation. At each time $t$, an individual with position $\theta$ at this time will turn to the right, that is to say, counterclockwise, or to the left, according to whether the proportion of individuals whose angle position is in the right half-plane ( $\theta \theta, \theta+\pi[$ ) is bigger than the one in the left half-plane, or the contrary. This term could also be interpreted as a correction made by the fish to the dispersion effect.

The parameter $\beta$ can be viewed as an intensity factor which sets up the strength of that correction. We have performed the study in terms of the parameter $\lambda=\beta / d$. It was shown that stability is lost as $\lambda$ crosses 
a certain threshold $\lambda_{\mathrm{c}}(=\pi / 2)$ and a set of nontrivial steady states emerges near this value. In fact, below that value $\lambda_{\mathrm{c}}$, dispersion dominates and the population organizes itself asymptotically as if there were no gregarism (Adioui et al., in press). When the ratio $\lambda$ exceeds $\lambda_{\mathrm{c}}$, the group starts to acquire a certain polarization. We have also proven in Adioui et al. (in press) that the local branch can be extended to a larger branch which is unbounded in $\lambda$, so that for each $\lambda>$ $\lambda_{c}$, there exists a nontrivial steady-state. Describing the evolution of the steady-state as $\lambda$ becomes large remains an open question.

\section{Lagrangian alignment model}

\subsection{IBMs in ecology}

We now present the Lagrangian model introduced in this study and the IBM arising from it, that is its computer simulation apparatus. In contrast with the Eulerian approach which looks at fluxes, a Lagrangian model addresses the level of individual organisms and describes changes in the state of individuals (here, it is just the angular position) as a result of forces acting on them. In recent years, a number of researchers have developed IBMs of fish schooling behavior to investigate which movement rules are required to produce cohesive schools capable of directional movement (see Niwa, 1996; Huth and Wissel, 1994; Lorek and Sonnenschein, 1998, for a review).

However, the subject did not really attract much interest until the beginning of the 1990s where, probably stimulated by the availability of powerful personal computers, it started to develop both at theoretical (foundations) and application levels (see DeAngelis and Gross, 1992, for a review).

Much of the popularity of IBMs stems from their ability to "imitate" real populations: in particular, each individual within a population is unique and differs from others in many biological respects (DeAngelis and Gross, 1992). Another reason for IBM's growing use amongst modelers is that important features of individuals are not taken into account in state variable models (e.g. individual variability) (Grimm, 1999), while, in contrast, IBMs are based on a somewhat detailed description of the behavior of individuals and their interactions with each other (DeAngelis and Gross, 1992). All individuals of a population may have different attributes like age, weight or position in an area that may influence the behavior of individuals (Gronewold and Sonnenschein, 1998).

Let us briefly recall that the principle of an IBM consists in following each individual of a collection which has been "seeded" at the beginning of the "virtual" experiment, assuming they move or some of their characteristics change during a time step, due to a number of influences, namely (Mogilner et al., 2003):

(i) individual behavior (for example, ability to move, specific speed, changes in internal state over time);

(ii) interactions of individuals with one another (mutual attraction or avoidance, repulsion at high densities, etc.);

(iii) interactions of individuals and a collection of individuals with a resource distribution, other species, or other aspects of the environment.

\subsection{A Lagrangian alignment model}

Based on the principles we just recalled, we built up a Lagrangian model, as follows. We assume that we work with $N$ individuals $(N \in \mathbb{N}-\{0\})$, each of them having - at some time $t$ - a certain angular position: $\left(\theta_{N}^{k}(t)\right)_{1 \leq k \leq N}$ is the family of angles for the $N$ individuals. Suppose that each $\left(\theta_{N}^{k}(t)\right)_{t \in \mathbb{R}^{+}}$is a stochastic process.

The Lagrangian alignment model is given via a system of stochastic differential equations as follows:

$d \theta_{N}^{k}(t)=F\left[\left(\theta_{N}^{l}(t)\right)_{1 \leq l \leq N}\right]\left(\theta_{N}^{k}(t)\right) d t+[\sqrt{2 d}] d W^{k}(t)$, $k=1, \ldots N$,

where we assume the angular position of the $k$ th individual is subject to random dispersal modeled as a Brownian distribution $W^{k}$, with diffusion parameter $d$. The drift term $F$ describes the alignment interaction rule which assures that fish tend to align in the direction of the average flux in their vicinity and the strength of this force is determined by the term $\beta$ :

$F=\beta\left(\frac{v^{1}-v^{2}}{N}\right)$, 
with

$v^{1}(\theta)=\sum_{l=1}^{N} 1_{]-\pi, 0]}\left(\theta-\theta_{N}^{l}(t)\right)$,

$v^{2}(\theta)=\sum_{l=1}^{N} 1_{] 0, \pi]}\left(\theta-\theta_{N}^{l}(t)\right)$.

$1_{]-\pi, 0]}$ (resp. $1_{0, \pi]}$ ) is the indicator function of the interval $]-\pi, 0]$ (resp. ]0, $\pi]$ ) $1_{]-\pi, 0]}(\theta)=1$ if $\theta \in$ ]$-\pi, 0]$, and 0 if else. Using (2), we get

$\frac{v^{1}-v^{2}}{N}=G \times \frac{\sum_{l=1}^{N} \delta_{\theta_{N}^{l}(t)}}{N}$,

that is

$$
\begin{aligned}
& F\left[\left(\theta_{N}^{l}(t)\right)_{1 \leq l \leq N}\right]\left(\theta_{N}^{k}(t)\right) \\
& \quad=\beta\left(G \times \frac{\sum_{l=1}^{N} \delta_{\theta_{N}(t)}}{N}\right)\left(\theta_{N}^{k}(t)\right),
\end{aligned}
$$

where $\delta_{\theta_{N}(t)}$ denotes the Dirac measure at point $\theta_{N}^{l}(t)$.

\subsubsection{From microscopic rules to a macroscopic description}

The passage from the "microscopic" Lagrangian description, to the "macroscopic" Eulerian one (1) is undertaken by letting $N$ go to infinity in the above description. So doing, the empirical distribution $\theta_{N}(t)=(1 / N) \sum_{k=1}^{N} \delta_{\theta_{N}^{k}(t)}$ converges to a probability density $U(\theta, t), \beta[G \times U(\cdot, t)](\theta)$ is the limit of the right-hand side of (3). We refer to Capasso (2000), Friedman (1975) and Metivier (1968) for further information on this subject.

\subsection{The simulator and scenarios tested}

The simulator is provided with an operator interface, intended for real time and easy-to-perform action-to-visualize alignment behavior. It is implemented in JAVA (Jdk 1.1.8, SunMicrosystems), an object-oriented program language. In an objectoriented system, a class is a collection of data and methods that operate on the data. Taken together, the data and methods describe the state and the behavior of an object. The interface consists of three components: (i) the parameter window, that allows to set up global variables (notably, $d$ : diffusion parameter $\left(\mathrm{rad}^{2} / \mathrm{s}\right), \beta$ : alignment parameter $(\mathrm{rad} / \mathrm{s}), N$ : number of individuals);

(ii) the situation window, that represents angular positions of individuals. It allows to observe the situation of fish's angles;

(iii) the indicator window that allows to visualize the evolution of the angular width of the distribution of individual fish angles.

In Section 2, we have recalled from Adioui et al. (in press) that the parameter $\lambda$, expressed as the ratio of the alignment parameter $\beta$ to the diffusion parameter $d$ plays an important role in the alignment phenomenon. Concretely, when the ratio $\lambda$ exceeds some threshold value $\lambda_{c}$, the group starts to be somehow polarized; below that value, dispersion dominates. As already mentioned, the conclusions drawn from the analytical study are severely limited to the immediate vicinity of $\lambda_{\mathrm{c}}$ and it was notably impossible to deduce from mathematical analysis strong alignment, meaning tight angular width with all individuals heading roughly in the same direction (Adioui et al., in press).

The approach proposed here, that is numerical simulations, was undertaken with the double goal of exploring such properties and comparing these with theoretical results, on the one hand, and, on the other hand, complementing the theoretical approach, by extending the range of parameters to be visited, for example large values of $\lambda$, and estimating further relevant quantities, such as the time of stabilization.

In view of this, we are therefore going to consider several experiments corresponding to different values of $d, \lambda$, and $N$. Each scenario is characterized by a single set of values for the parameters $(d, \lambda, N)$.

Outputs consist of:

(i) the standard deviation (the angular width) of the distribution of angular positions at the steady-state (defined as any distribution of the angular positions that is unaffected by simulation runs).

(ii) the stabilization time, the time $\tau$ after which steady-state is achieved. Note that the average angular position is not a relevant quantity, since the space is considered isotropic. In fact, different experiments will generally lead to different averages, which are likely to change at each run, even at a steady state. 
By preliminary experimental studies, it was shown that some variability occurs in the outputs, on the standard deviation as well as on the stabilization time. For that reason, we perform five runs of the simulator, for each scenario. This number of runs appears to be sufficient to damp out randomness embodied in the simulator. In Mogilner et al. (1999), the results of the numerical experiments describing the evolution of propagating pulses were plotted three times.

Preliminary results have also shown that $1000 \mathrm{~s}$ of simulated time is a sufficient long time to reach a steady state distribution, with a time step less than or equal to $0.1 \mathrm{~s}$.

We perform our results for three values of $d$ ( $d=$ $0.011,0.11,1.1)$ corresponding to three different orders of magnitude. Another aspect is the effect of the school size. We compare two sizes: $N=100$ and 1000. The simulations presented here were obtained with the uniform distribution, as the initial distribution.

\section{Simulation results}

In this section, we present the main results obtained in simulations.

\subsection{Result 1 (threshold)}

The theoretical study performed in Adioui et al. (in press) leads to existence of a non-uniform distribution for all $\lambda$ larger than some critical value $\lambda_{\mathrm{c}}(=\pi / 2)$. The stability of the solution, however, could only be ascertained in the vicinity of $\lambda_{\mathrm{c}}$. Further solutions could also emerge away from $\lambda_{\mathrm{c}}$. We are not able to derive such solutions by theoretical computation.

\subsubsection{Transitional dynamics}

In accordance with what was found from the mathematical analysis, we want to test a "numerical" threshold value of $\lambda$ in the vicinity of which there is a sharp change in stability. Concentrating on the standard deviation data obtained by simulation, we see that for $\lambda \in[0,1.5]$, the standard deviation is first constant and then goes down slowly, with a total relative variation less than $20 \%$. Beyond the value 1.5 , the value of the standard deviation goes down faster and loses $85 \%$ when $\lambda$ increases from 1.5 up to 2.0. The value 1.5 is in surprisingly close agreement with $\lambda_{c}$ considering the erratic nature of the simulated data. Experiments shown in Fig. 1 demonstrate that the degree of alignment is controlled by a parameter $\lambda$ : for $\lambda=0$ there is no alignment at all, no structuration can be observed; as $\lambda$ reaches the value 1.5 , a dynamical phase transition exhibiting spontaneous breaking of diffusive behavior occurs, and therefore aligned patches start to form.

\subsubsection{Perfect alignment}

We also investigated the dynamics of the system away from the vicinity of the bifurcation point $\lambda_{c}$, in a range of the parameter values where the mathematical analysis is out of reach. Simulations undergone for values of $\lambda$ (any) larger than $\lambda_{\mathrm{c}}$ confirm the narrowing of the angular width: with all due reservations, the standard deviation approaches zero as in Fig. 2. So, increasing the parameter $\lambda$ leads to pattern formation manifested by collectively moving clusters consisting of fish having a similar angular position. However, for $d=1.1$, the adverse situation (that is the broadening of the angular width) does not occur beyond $\lambda \approx 6$ (see Fig. 2). Computer simulations (which we do not present here) show that when $\beta$ exceeds the value 6.0 approximately, this property holds true also independent on the value of $d$.

\subsection{Result 2 (time of stabilization)}

We examined the time of stabilization, that is the time it takes to reach a steady-state. We summarize our findings in the following remarks and in Fig. 3:

(i) $1000 \mathrm{~s}$ is a time long enough to reach a steady-state.

(ii) The time of stabilization is at its maximum when $\lambda$ is close to the bifurcation point $\lambda_{c}$.

(iii) For values of $\lambda$ (any) larger than $\lambda_{c}$, the time of stabilization decreases when $\lambda$ increases.

(iv) For $\lambda$ fixed, the time of stabilization decreases as $d$ increases.

These results can be interpreted as: when $\lambda$ crosses a critical value $\lambda_{\mathrm{c}}$ upwards, while remaining close to it, two steady states take place: a trivial one, weakly unstable, and a non-trivial steady state, weakly stable. Consequently the time of stabilization necessary for an initial data close to the bifurcation point $\lambda_{c}$, to 

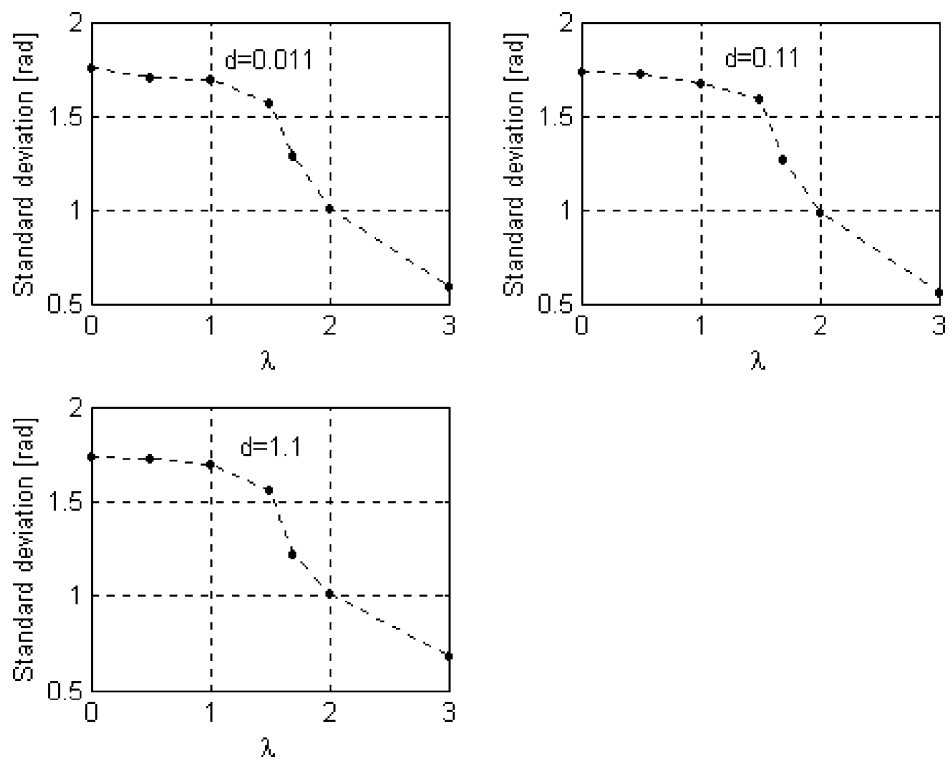

Fig. 1. Phase diagram for alignment interaction. Shown are the regions of stable and unstable, as a function of $\lambda$ for $d=0.0110 .11,1.1$. These figures were obtained after $1000 \mathrm{~s}$ for groups of size $N=100$.

go away from the trivial steady-state weakly unstable to the non-trivial steady-state weakly stable becomes large.

In other words, the time required for dynamical transition from unordered state to alignment is large close to $\lambda_{\mathrm{c}}$. In contrast, for $\lambda$ far enough from $\lambda_{\mathrm{c}}$, the alignment behavior occurs rapidly and then the time of stabilization decays gradually to zero. The situation (iv) is rather largely different in the case when $\lambda$ is close to $\lambda_{\mathrm{c}}$. Comparing the case of $d=0.011,0.11$
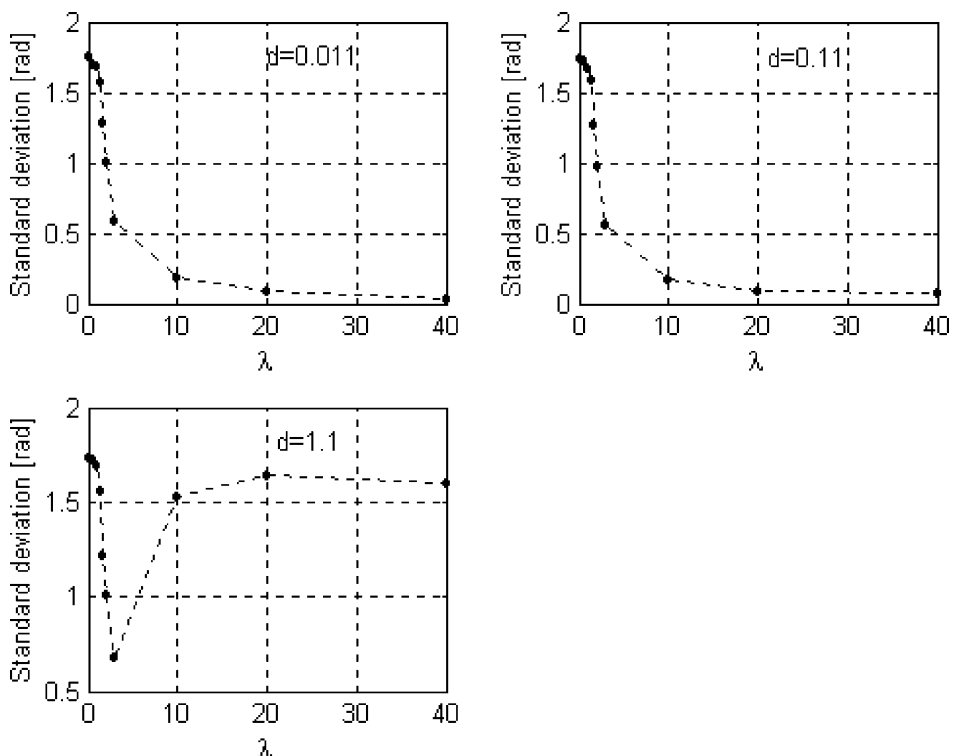

Fig. 2. Standard deviation vs. parameter $\lambda$. These values were obtained after $1000 \mathrm{~s}$, for groups of size $N=100$ for $d=0.011,0.11,1.1$. 

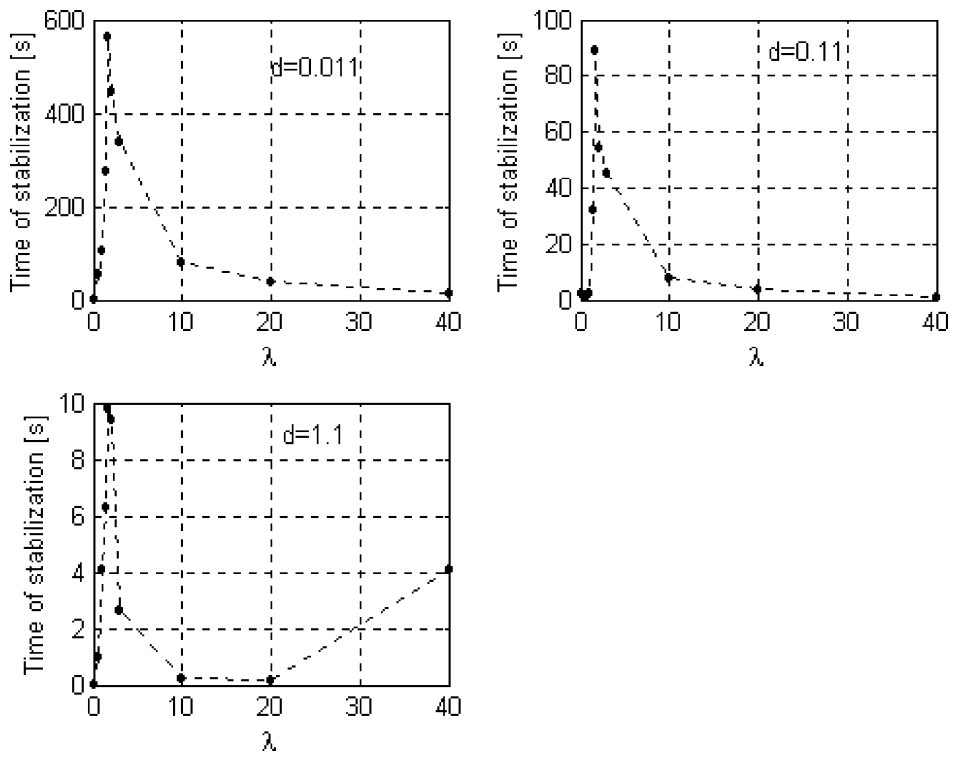

Fig. 3. Time of stabilization vs. $\lambda$ for $d=0.011,0.11,1.1$ after $1000 \mathrm{~s}$, for groups of size $N=100$.

versus 1.1 , the corresponding time of stabilization (in s) is $564.555,89.301$ versus 9.834 , for parameter $\lambda \approx 1.7$.

\subsection{Result 3 (effect of density)}

Another important question is, are simulations also valid for bigger schools? In other words, is there a lower or an upper limit for the simulations to be affected by the size of the school?

A thorough treatment of this issue would require a considerable number of experiments. Our result is just illustrative: we compared two cases, $N=100$ and 1000.

\subsubsection{Standard deviation}

The simulations presented earlier were performed for schools of 100 individuals. When the size of the group is very large, that is if we increase the number of school members (beyond 100), we get the same results (Fig. 4). As shown in Fig. 1, two plausible scenarios for the transition can be proposed: non-cooperative, so that the angular distribution of individuals is nearly uniform on the one hand, and, on the other hand, the cooperative one, in which individuals display schooling behavior. Fig. 4 shows that with the same parameter $\lambda$, the values of standard deviation corresponding to $N=100$ and 1000 are nearly similar. This result suggests that the group maintains its structure while the number of organisms increases. In other words, the dispersion of orientations is not affected by the density: increasing or decreasing the number of individuals with the same parameter $\lambda$ does not change the standard deviation.

\subsubsection{Time of stabilization}

In Fig. 5, we explore the time of stabilization in terms of the density of the individuals. Experiments shown in Fig. 5 demonstrate that the time of stabilization depends mainly on the size of the school. This dependence is high enough in the vicinity of $\lambda_{\mathrm{c}}$. In fact, comparing the case of $N=100$ and 1000, the corresponding time of stabilization (in s) is $\mathbf{5 6 4 . 5 5 5}$ and 929.611, respectively, for parameters $\lambda=1.7$ and $d=0.011$ (see Fig. 5). This means that the stabilization time of transitional dynamics of the angular distribution from angular disorder to alignment is very important for large groups. For values of $\lambda$ much larger than $\lambda_{\mathrm{c}}$, the stabilization time decays rapidly to zero for $N=100$ as well for $N=1000$. Furthermore, the remarks made in the case of $N=100$ (see Section 4.2) hold true also for $N=1000$. 

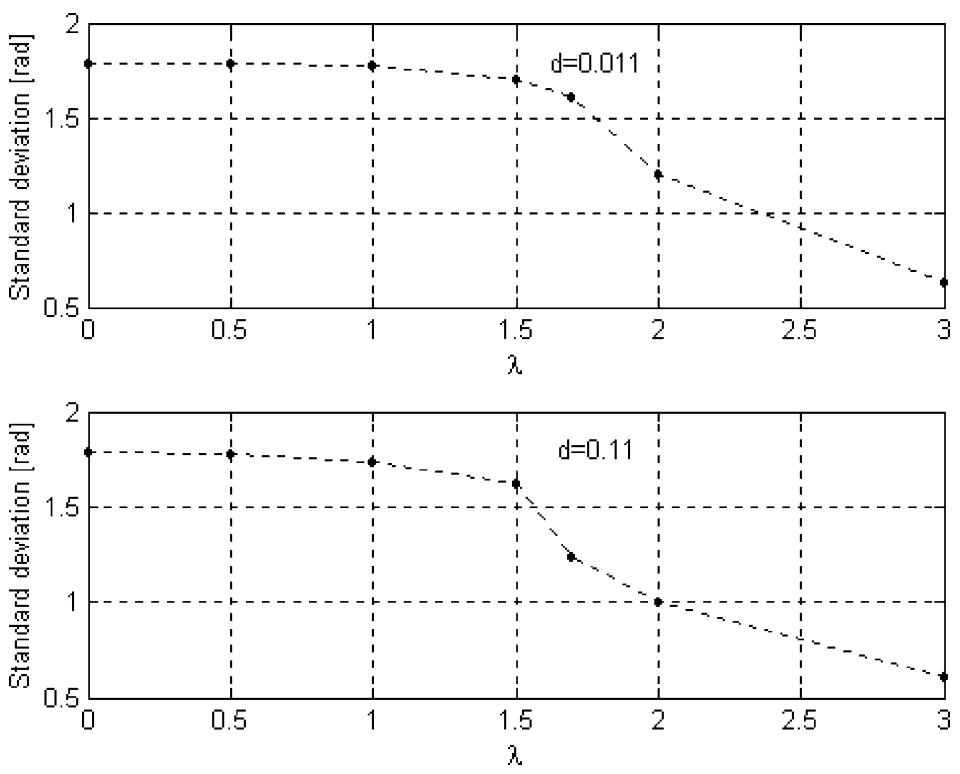

Fig. 4. Phase diagram for alignment interaction. Shown are the regions of stable and unstable, as a function of $\lambda$ for $d=0.011,0.11$. These graphs were obtained after $1000 \mathrm{~s}$, for groups of size $N=1000$.

\subsubsection{Variability}

The analysis of the outputs for each simulation shows that some variability occurs, on the standard deviation as well as on the stabilization time. We can see that the variability is at a maximum in the vicinity of $\lambda_{\mathrm{c}}$, and then decreases rapidly as $\lambda$ increases (Fig. 6). Fig. 6 also shows that the variability of standard deviation depends on the size of the population. In fact,
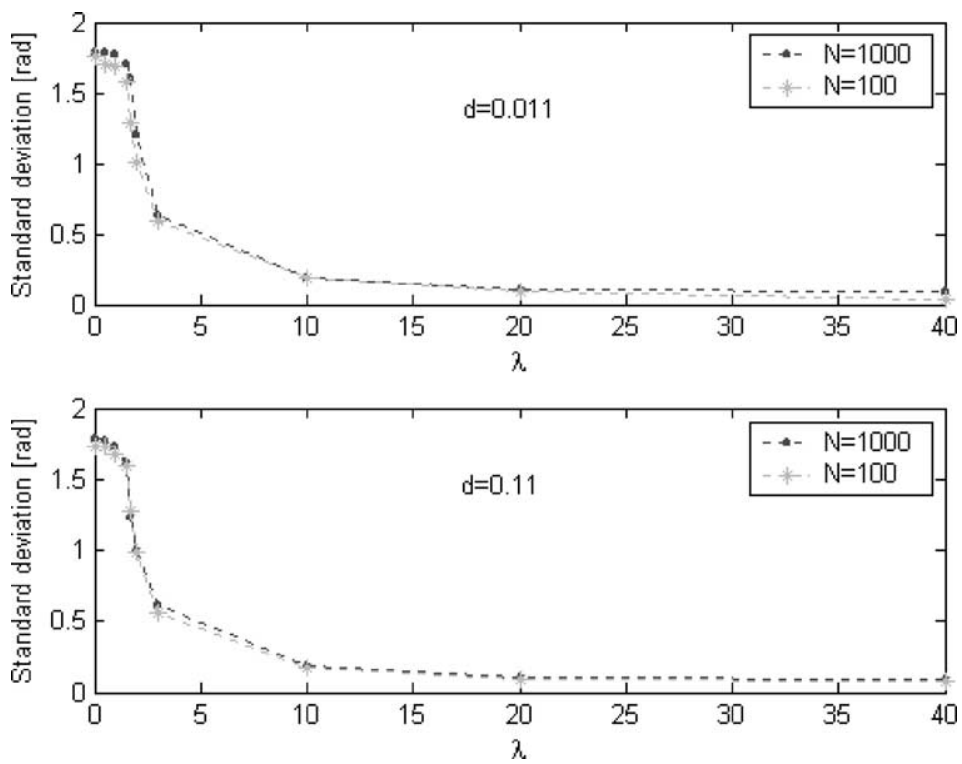

Fig. 5. Comparison of standard deviation vs. parameter $\lambda$ between the groups of size $N=100$ and the groups of size $N=1000$. These values were obtained after $1000 \mathrm{~s}$ for $d=0.011,0.11$. 

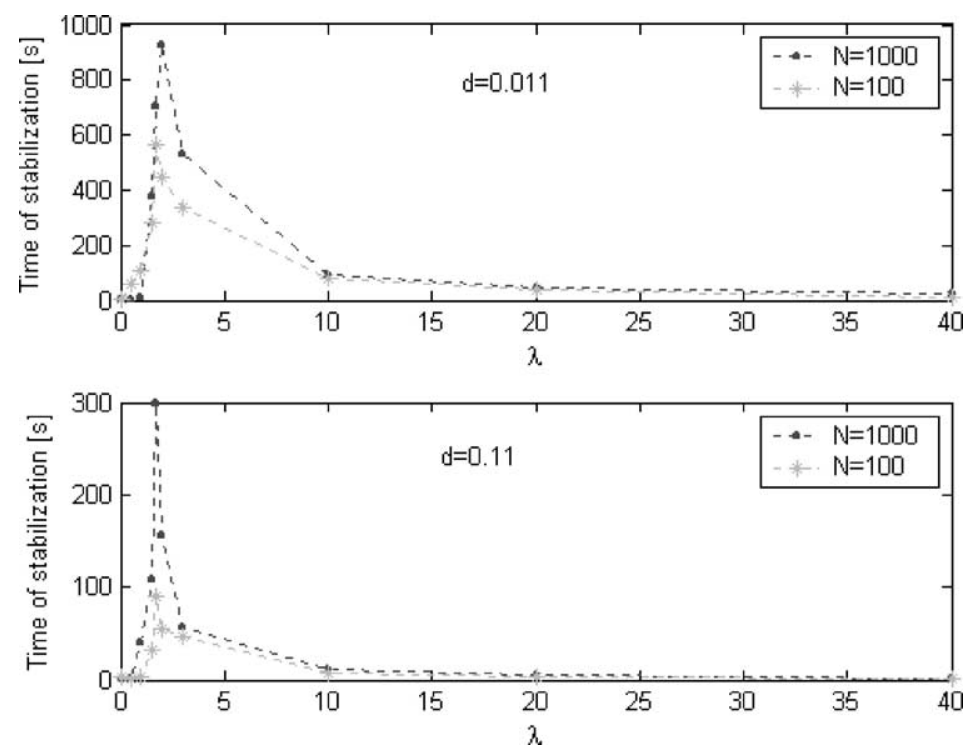

Fig. 6. Comparison of time of stabilization vs. parameter $\lambda$ between the groups of size $N=100$ and the groups of size $N=1000$. These values were obtained after $1000 \mathrm{~s}$, for $d=0.011,0.11$.

we can remark that the variability of standard deviation corresponding to $N=100$ is greater than the one for $N=1000$. This difference reaches its maximum in the vicinity of $\lambda_{c}$. In contrast, the variability of the time of stabilization is higher for $N=1000$ than for $N=100$, especially in the vicinity of $\lambda_{\mathrm{c}}$ for $d=0.11$ (Fig. 6).

\section{Conclusion}

In this paper, a mixed Lagrangian-Eulerian approach was undertaken in the study of the alignment process in a fish school. Most of the paper was devoted to the IBM; an earlier work done on a PDE arising from the Eulerian approach was presented elsewhere (Adioui et al., in press). The PDE was used to calibrate the IBM on the value of a threshold, which was only imperfectly reproduced by the IBM. In this sense, the approach is really mixed.

The work was done under simplifying assumptions which notably allow us to concentrate on the competition between alignment and dispersion and discard the other individual forces of attraction and repulsion. Both the Eulerian model and the IBM are rather simple ones and we do not suggest that real animals will adhere strictly to them. We were interested in exploring how a spontaneous preferred orientation may arise, what types of interactions promote this kind of pattern formation, and under what conditions. The alignment response forms the main phenomenon of interest here.

We performed our results for three values of the diffusion coefficient $d\left(\mathrm{rad}^{2} / \mathrm{s}\right), d=0.011,0.11,1.1$. The corresponding statistical dispersion of angular position (in ${ }^{\circ}$ ), at successive time intervals of length $0.01 \mathrm{~s}$, is $0.8498,2.6874,8.4983$, respectively. In the literature (Huth and Wissel, 1994; Mikhailov and Calenbuhr, 2002), the parameter which controls dispersion of angular position at $0.5 \mathrm{~s}$ is equal to $15^{\circ}$, so it leads to a value of $d=0.0685\left(\mathrm{rad}^{2} / \mathrm{s}\right)$. This typical value is in the range of orders of magnitude of $d$ taken in our investigation.

Polarization $P$ is frequently defined as the average of the angle deviation of each fish to the mean swimming direction of the school. Here, it is the standard deviation of the angular position (no swimming is involved during the alignment process). The values of the parameter $\lambda$ used in the experiments, that is $\lambda \in[0,40]$ are sufficient to reproduce the high levels of polarization reported by some authors (Huth and Wissel, 1994; Mikhailov and Calenbuhr, 2002). 
For example, mackerel schools show the highest parallel orientation $\left(P \in\left[8,12^{\circ}\right]\right)$ (Huth and Wissel, 1994). With our simulation model, we can reproduce these values of $P$ by changing the range of $\lambda$. For example, as $\lambda$ is increased from 5 to $20, P$ decreases accordingly from $20^{\circ}$ to approximately $5^{\circ}$. A crucial assumption of our model is that fish are at rest with respect to one another. In fact, a more useful view would be to assume that alignment is taking place in a moving school where mutual distances may change with time, but it acts much faster than the other processes. Suppose for example a school moving at a speed $v$ with fish heading possibly at different directions.

Let $t_{v}$ be the time necessary for the distance of two fish to go from $1 \mathrm{BL}$ to over $5 \mathrm{BL}$, which we assume to be the maximal distance of perception. Denoting $\alpha$ their mutual angle, it is a matter of easy calculation to get that $t_{v}=2 / v \sin (\alpha / 2)$. Estimating $\alpha$ as equal $2 P$, we obtain a mean value estimate of the time it takes to two fish to be out of mutual perception, just as a result of drift, namely, $t_{v}=2 / v \sin (P)$. A typical value of the maximal velocity of a fish is $1.3 \mathrm{BL} / \mathrm{s}$ (Huth and Wissel, 1994). In order for the alignment process to succeed, it is thus necessary that the stabilization time $\tau$ be much less than $t_{v}$. The data obtained by our experiments show that this phenomenon is reproduced if, $P \approx 2^{\circ}$ (corresponding to $\lambda=40$ ). Indeed, for $P \approx$ $2^{\circ}, t_{v}=38 \mathrm{~s}$ and $\tau=12 \mathrm{~s}$, so we have $\left(\tau<t_{v}\right)$. In the case of $P \approx 10^{\circ}$ (corresponding to $\lambda=10$ ), however, we have (for $v=v_{\max }$ ) $\tau=80.624 \mathrm{~s}$, far too much above $t_{v}=8.3763 \mathrm{~s}$. According to the formula $v=$ $2 \mathrm{BL} / t_{v} \sin (P)$, such a polarization $P \approx 10^{\circ}$ requires $v<0.135 \mathrm{BL} / \mathrm{s}$, that is for $v=v_{\max } / 10$.

Compared to the literature, our results overestimate the polarization that is needed for a school to persist at a given speed, or, at a given polarization, the speed should be lower than in the literature (Mikhailov and Calenbuhr, 2002). The orders of magnitude though are not so incredibly different, if we take into account the fact that the model used here is really a very basic one: the force of alignment is certainly at fault by giving the same importance to all neighbors of a fish independently on their angular distance to it. Also, in all experiments the initial state is the uniform distribution: we did not account for a possible initial dispersion width which in general will be less than the maximum provided by the uniform distribution.
Otherwise, when the group is not polarized, the velocity of fish must be negligible in order to reform into a polarized school. This result agrees with the findings in Huth and Wissel (1994) and Mikhailov and Calenbuhr (2002). So, schools with a high parallel orientation find food patches better, because they are highly mobile.

One of the main qualitative results of this work is that bifurcation from angular disorder to alignment occurs when the ratio $\lambda$ of controlled to uncontrolled turning is greater than some critical value. Both simulations and mathematical analysis show that the distribution of angular positions of a fish group undertakes a continuous dynamical phase transition when $\lambda$ reaches a critical value, manifested by a change from dispersion to alignment, that is for $\lambda \in[0,1.5]$, the standard deviation is first slightly constant and then goes down slowly, with a total relative variation less than $20 \%$. The two markedly different distributions could be associated with two distinct behaviors that a group of fish would be able to display and the transition could correspond to the passage from one to the other according to a variety of circumstances.

Hara (1985) observed such transitions in the ocean: Japanese sardine groups which where performing amoebic movements at the beginning of observations were gradually drawn up in order and thus the groups would perform rectilinear movements. This behavior is also reproduced if, when a fish is surrounded by food, it loses its polarization and each fish snaps at food on its own. After the food patch has been consumed, the school reforms (Mikhailov and Calenbuhr, 2002). These observations display existence of two types of basic behaviors: shoal (fish which are randomly oriented within a group and are weakly polarized) and school (a group of fish that swim in a synchronized manner, i.e. with similar speeds and direction (highly polarized)) (Fréon and Misund, 1999). This suggests that the parameter $\lambda$ could in fact be a function of the satiation state of the group or some other time dependent variable, associated with the group. Work in this direction is highly desirable.

Another aspect treated in this paper is the effect of the parameter $\lambda$ on the time of stabilization: it is higher for $\lambda$ close to $\lambda_{\mathrm{c}}$ and then decreases as $\lambda$ increases (Figs. 7 and 8). We have also shown the influence of 

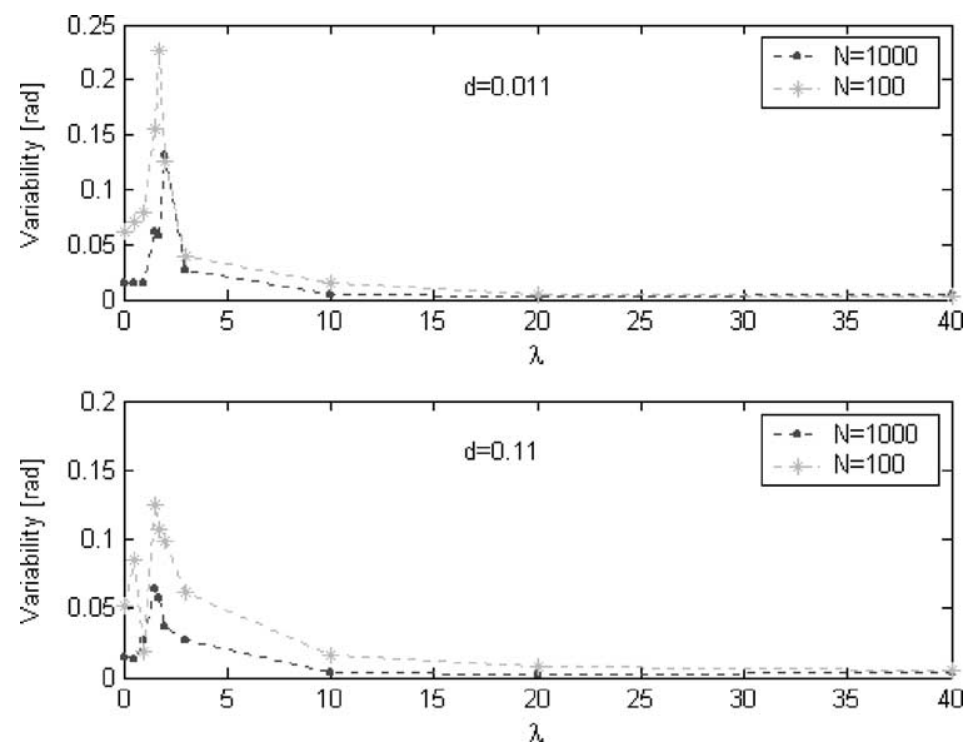

Fig. 7. Comparison of variability of standard deviation vs. parameter $\lambda$ between the groups of size $N=100$ and the groups of size $N=1000$. These values were obtained after $1000 \mathrm{~s}$, for $d=0.011,0.11$.

density on the dispersion of orientations as well as on the stabilization time (Section 4.3).

A sort of paradoxical result that we found, not included in the section of results, is that the angular width ceases to diminish for large values of $\beta$. This result, rather surprising if we keep in mind the significance of $\beta$, can in fact be interpreted by saying that, if the individual reactions of fish are too fast, they tend to act in anticipation of the alignment force and thus indirectly enhance the noise. One should also point
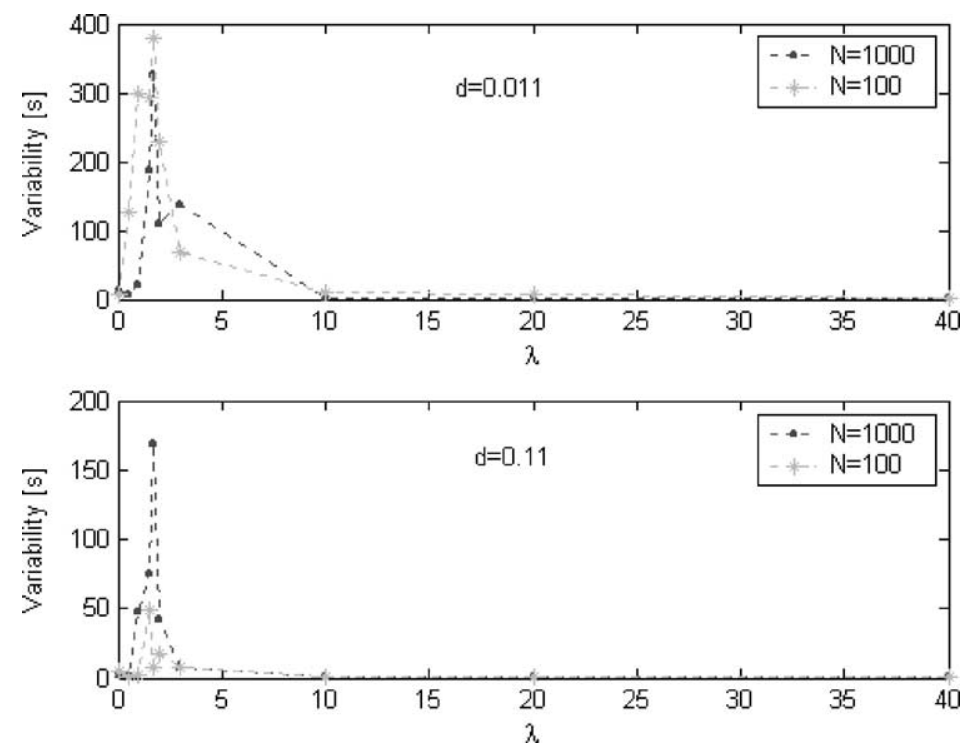

Fig. 8. Comparison of variability of time of stabilization vs. parameter $\lambda$ between the groups of size $N=100$ and the groups of size $N=1000$. These values were obtained after $1000 \mathrm{~s}$, for $d=0.011,0.11$. 
out that part of this effect may be due to the method used in implementing the computer simulation, that is synchronous simulation. Asynchronous computations might be more robust than the synchronous ones to artificial oscillations introduced by the numerical approximation, and could possibly extend the polarizing effect of $\beta$ a little further than our actual findings.

Finally, we would like to comment about the assumption in both the Lagrangian and the Eulerian models that a large number of fish, or even an infinite number of fish in the Eulerian model, influence the orientation of a given fish. In fact, a fish will most probably only look directly at a low number of its neighbors, the nearest ones in its sight's cone. So, if we consider a time step close to the reaction (time) to nearest neighbors, the assumption is certainly not correct. But, if we assume that the reaction of a fish to its nearest neighbors is much faster than the time step of the simulation, then the position taken by a fish after one simulation run will indirectly reflect the positions of the neighbors of its neighbors, of the neighbors of the neighbors of the neighbors, etc. This would define different layers of neighbors, each layer acting at a distinctive intensity, dependent upon the distance to the fish. The situation considered in this work could correspond to 10 nearest neighbors and two layers, therefore roughly the same intensity for the two layers, leading to the model of the paper.

\section{References}

Adioui, M., Arino, O., Smith, W.V., Treuil, J.P., in press. A mathematical analysis of a fish school model. J. Differ. Equations.

Alt, W., 1997. Dynamics of cell and tissue motion. In: Proceedings of Workshop Bonn-Röttgen Birkhäuser, Basel.

Aoki, I., 1980. An analysis of the schooling behavior of fish: internal organization and communication process. Bull. Ocean. Res. Inst. No. 12, Tokyo.

Aoki, I., 1982. A simulation study on the schooling mechanism in fish. Bull. Jpn. Soc. Fish No. 48, pp. 1081-1088.

Breder, C.M., 1959. Studies on the social groupings of fishes. Bull. Museum Am. Nat. History 117, 393-482.

Bumann, D., Krause, J., Rubenstein, D.I., 1997. Mortality risk of spatial positions in animals groups: the danger of being in the front. Behavior 134, 1063-1076.

Capasso, V., 2000. Stochastic differential equations and systems of integrating particles. Lecture notes in 1st ESMTB, Martina Franca (Taranto), Italy, pp. 4-15.
Deutsch, A., 1995. Orientation-induced pattern formation: swarm dynamics in a lattice-gas automation model. Int. J. Bifurc. Chaos 6, 1735-1752.

DeAngelis, D.L., Gross, L.J., 1992. Individual-Based Models and Approaches in Ecology, Populations, Communities and Ecosystems. Routledge, Chapman and Hall, New York.

Edelstein-Keshet, L., Ermentrout, G.B., 1990. Models for contact mediated pattern formation. J. Math. Biol. 29, 32-58.

Fréon, P., Misund, O.A., 1999. Dynamics of Pelagic Fish Distribution and Behavior: Effects on Fisheries and Stock Assessment. Fishing New Books.

Friedman, A., 1975. Stochastic Differential Equations and Applications, vols. I and II. Academic Press, London.

Grimm, V., 1999. Ten years of individual-based modelling in ecology: what have we learned and what could be learned in the future. Ecol. Model. 115, 129-148.

Gronewold, A., Sonnenschein, M., 1998. Event-based modelling of ecological systems with asynchronous cellular automata. Ecol. Model. 108, 37-52.

Grunbaum, D., 1994. Translating stochastic density-dependent individual behavior with sensory constraints to an Eulerian model of animal swarming. J. Math. Biol. 33, 139-161.

Grunbaum, D., 1998. Schooling as strategy for taxis in a noisy environment. Evol. Ecol. 12, 503-522.

Grunbam, D., Okubo, A., 1994. Modelling social animal aggregations. In: Levin, S.A. (Ed.), Frontiers in Mathematical Biology. Lecture Notes in Biomathematics, vol. 100. Springer-Verlag, Berlin, pp. 296-325.

Gueron, S., Levin, S.A., 1995. The dynamics of group formation. Math. Biosci. 128, 243-264.

Gueron, S., Levin, S.A., Rubenstein, D.I., 1996. The dynamics of herds: from individuals to aggregations. J. Theor. Biol. 182, 85-98.

Hara, I., 1985. Moving direction of Japanese sardine school on the basis of aerial surveys. Bull. Jpn. Soc. Sci. Fish. 51, 19391945.

Huth, A., Wissel, C., 1994. The simulation of fish schools in comparison with experimental data. Ecol. Model. 75, 135145.

Huston, M., DeAngelis, D., Post, W., 1988. New computer models unify ecological theory. BioScience. 38, 682-691.

Krause, J., Hoare, D., Krause, S., Hermelrijk, C.K., Rubenstein, D.I., 2000. Leadership in fish shoals. Fish and Fisheries 1, 8289.

Lorek, H., Sonnenschein, M., 1998. Object-oriented support for modelling and simulation of individual-oriented ecological models. Ecol. Model. 108, 77-96.

Metivier, M., 1968. Notions Fondamentales de la Théorie des Probabilités. Dunod, Paris.

Mikhailov, A.S., Calenbuhr, V., 2002. From cells to societies, Springer Series in Synergetics, June.

Mogilner, A., Bent, L., Edelstein-Keshet, L., 2003. An individual-based model for aggregation in social organisms: attraction-repulsion interactions that lead to a well-spaced group. In press.

Mogilner, A., Bent, L., Edelstein-Keshet, L., 1996. Spatio-angular order in populations of self-aligning objects: formation of oriented patches. Physica D 89, 346-367. 
Mogilner, A., Bent, L., Edelstein-Keshet, L., 1999. A non-local model for a swarm. J. Math. Biol. 38, 534-570.

Niwa, H., 1996. Newtonian dynamical approach to fish schooling. J. Theor. Biol. 181, 47-63.

O'Brien, D.P., 1989. Analysis of the internal arrangement of individuals within crustacean aggregations. J. Exp. Mar. Biol. Ecol. 124, 1-30.

Parrish, J.K., Edelstein-Keshet, L., 1999. Complexity, pattern and evolutionary trade-offs in animal aggregation. Science 284,99 101.

Parrish, J.K., Hamner, W.M., 1997. Animals Groups in Three Dimensions. Cambridge University Press, Cambridge.
Pitcher, T.J., Parrish, J.K., 1986. Functions of shoaling behavior in teleosts. In: Pitcher, T.J. (Ed.), The Behavior of Teleost Fishes. Chapman and Hall, London, pp. 294-337.

Reynolds, C.W., 1999. Flocks, herds and schools: a distributed behavior model. Comput. Graph. 21 (1987), 25-34.

Stöcker, S., 1999. Models for tuna school formation. Math. Biosci. 156, 167-190.

Vabø, R., Nøttestad, L., 1997. An individual model of fish school reactions: predicting antipredator behavior as observed in nature. Fish Oceanogr. 6, 155-171.

Warburton, K., Lazarus, J., 1991. Tendency-distance of social cohesion in animal groups. J. Theor. Biol. 150, 473-488. 